\title{
Exact Tagged Particle Correlations in the Random Average Process
}

\author{
R. Rajesh ${ }^{1}$ and Satya N. Majumdar ${ }^{1,2}$ \\ 1. Department of Theoretical Physics, Tata Institute of Fundamental Research, Homi Bhabha Road, Mumbai 400005, India. \\ 2. Laboratoire de Physique Quantique (UMR C5626 du CNRS), Université Paul Sabatier, 31062 Toulouse Cedex, France.
}

(October 30, 2018)

\begin{abstract}
We study analytically the correlations between the positions of tagged particles in the random average process, an interacting particle system in one dimension. We show that in the steady state the mean squared auto-fluctuation of a tracer particle grows subdiffusively $\sigma_{0}^{2}(t) \sim t^{1 / 2}$ for large time $t$ in the absence of external bias but grows diffusively $\sigma_{0}^{2}(t) \sim t$ in the presence of a nonzero bias. The prefactors of the subdiffusive and diffusive growths as well as the universal scaling function describing the crossover between them are computed exactly. We also compute $\sigma_{r}^{2}(t)$, the mean squared fluctuation in the position difference of two tagged particles separated by a fixed tag shift $r$ in the steady state and show that the external bias has a dramatic effect in the time dependence of $\sigma_{r}^{2}(t)$. For fixed $r, \sigma_{r}^{2}(t)$ increases monotonically with $t$ in absence of bias but has a non-monotonic dependence on $t$ in presence of bias. Similarities and differences with the simple exclusion process are also discussed.
\end{abstract}

PACS numbers: 64.60.-i, 05.70.Ln

\section{INTRODUCTION}

Interacting particle systems in one dimension are amongst the simplest examples of many body systems that are far from equilibrium [1]. One of the most studied examples is the simple exclusion process in one dimension. In this system, each site of a one dimensional lattice is either occupied by a hardcore particle or it is empty. In a small time interval $d t$, each particle attempts to hop to the neighboring lattice site on the right with probability $p d t$, to the left neighboring site with probability $q d t$ and stays at the original site with probability $1-(p+q) d t$. An attempted hop is completed provided the target site is empty. A wealth of results are known for this system [1] 3].

Another interacting particle system in one dimension that has attracted recent interest is the random average process (RAP) 田, D. In the RAP, particles are located on a real line as opposed to a lattice in the simple exclusion process. Let $x_{i}(t)$ be the position of the $i$-th particle at time $t$ (see Fig. 1).

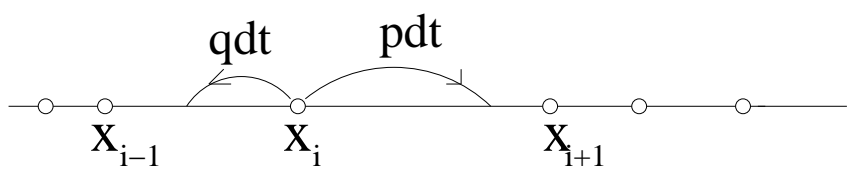

FIG. 1. The stochastic moves in the RAP.

In a small time interval $d t$, each particle jumps to the right with probability $p d t$ by an amount $r_{i}^{+}\left(x_{i+1}-x_{i}\right)$, to the left with probability $q d t$ by an amount $r_{i}^{-}\left(x_{i}-\right.$ $\left.x_{i-1}\right)$ and stays at its original location with probability $1-(p+q) d t$. Here $r_{i}^{+}$and $r_{i}^{-}$are independent random variables drawn from the interval $[0,1]$ with identical probability density function (pdf) $f(r)$. Thus the jumps in either direction is a random fraction of the gap to the nearest particle in that direction. For convenience, we have defined the RAP with random sequential dynamics, though it has been studied with parallel dynamics as well [4.5]. The detailed study of the RAP is important since it has shown up either directly or in disguise in a

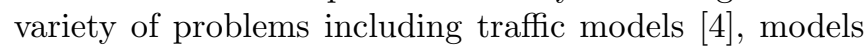
of mass transport [5], models of force fluctuation in bead packs [6], models of voting systems [7, 8], models of wealth distribution [9] and the generalized Hammersley process [10]. Like the simple exclusion process, some aspects of the RAP are analytically tractable [4. 5. 11]. In this paper, we derive some new exact results on the tracer fluctuations in the RAP where the dynamics of tagged particles are followed.

The tracer diffusion has been studied in detail for the simple exclusion process and many interesting results are known [1]. In the exclusion process, the combined effect of hardcore interaction and the external bias $(p-q)$ shows up rather dramatically in the asymptotic long time behavior of the mean squared auto-fluctuation in the position of a tracer particle in the steady state. If $\zeta_{i}(t)=x_{i}(t)-\left\langle x_{i}(t)\right\rangle$ denotes the deviation in the position $x_{i}(t)$ of the $i$-th particle from its average value, then the mean squared auto-fluctuation is defined as, $\sigma_{0}^{2}\left(t_{0}, t_{0}+t\right)=\left\langle\left[\zeta_{i}\left(t_{0}+t\right)-\zeta_{i}\left(t_{0}\right)\right]^{2}\right\rangle$, where $t_{0}$ is the waiting time after which one starts measuring the fluctuations. In the steady state $t_{0} \rightarrow \infty$, the asymptotic behavior of $\sigma_{0}^{2}(t)=\lim _{t_{0} \rightarrow \infty} \sigma_{0}^{2}\left(t_{0}, t_{0}+t\right)$ for large $t$ is known [1]. In absence of external bias $(p=q=1 / 2)$, i.e. for the symmetric exclusion process (SEP), $\sigma_{0}^{2}(t) \sim A t^{1 / 2}$ for large $t$ where the constant $A=(2 / \pi)^{1 / 2}(1-\rho) / \rho$ is known exactly in terms of the density $\rho$ of the particles 12214 . This slow subdiffusive growth is due to the caging effect arising from hard core exclusion in one dimension where 
a particle is always hemmed in by its neighbors. However in the asymmetric case (ASEP) when a nonzero bias $p-q>0$ is switched on, one finds, somewhat unexpectedly, $\sigma_{0}^{2}(t) \sim D t$ for large $t$ where the tracer diffusion coefficient $D=(p-q)(1-\rho)$ [15,16]. The crossover from the subdiffusive to diffusive behavior of $\sigma_{0}^{2}(t)$, as an infinitesimal bias is switched on, was understood in a physically transparent way via a rather unusual mapping of the exclusion process to a $(1+1)$-dimensional interface model [17, 18]. This mapping also established that an appropriately defined sliding tagged-particle correlation function varies anomalously as $t^{2 / 3}$ [17. This anomalous $t^{2 / 3}$ growth also shows up in the mean square fluctuation of the center of mass of the particles when viewed from a special moving frame 19].

A question then arises naturally: what are the corresponding results on the tracer diffusion for the RAP? The only known result is for the fully asymmetric RAP with $q=0$ (and time rescaled by $p$ ) where the particles move only to the right. In this limit, $\sigma_{0}^{2}(t)$ was computed by Krug and Garcia using a phenomenological hydrodynamic Langevin equation based on heuristic arguments as well as using an independent jump approximation [4]. Their result shows that $\sigma_{0}^{2}(t) \sim D_{1} t$ for large $t$ with $D_{1}=\rho^{-2} \mu_{1} \mu_{2} /\left(\mu_{1}-\mu_{2}\right)$ where $\rho$ is the density of the particles and $\mu_{k}=\int_{0}^{1} d r r^{k} f(r)$ is the $k$-th moment of the pdf $f(r)$. Later, Schütz attempted to derive this result rigorously 20] by writing down the exact equation of evolution of the equal time correlation function $G_{r}(t)=\left\langle\zeta_{0}(t) \zeta_{r}(t)\right\rangle$ and then using a chain of arguments. Note that the definition $\sigma_{0}^{2}\left(t_{0}, t_{0}+t\right)=\left\langle\left[\zeta_{i}\left(t_{0}+t\right)-\zeta_{i}\left(t_{0}\right)\right]^{2}\right\rangle$ involves both the variance $\left\langle\zeta_{i}^{2}(t)\right\rangle$ which is an equal time observable as well as the unequal time correlation $\left\langle\zeta_{i}\left(t_{0}\right) \zeta_{i}\left(t_{0}+t\right)\right\rangle$. Thus a proper approach, as followed in this paper, would be to compute these correlation functions exactly and then take the steady state $t_{0} \rightarrow \infty$ limit.

The main results of this paper can be summarized as follows:

1. We compute exactly the mean squared autofluctuation in the displacement of a single tracer particle, $\sigma_{0}^{2}\left(t_{0}, t_{0}+t\right)=\left\langle\left[\zeta_{i}\left(t_{0}+t\right)-\zeta_{i}\left(t_{0}\right)\right]^{2}\right\rangle$ for large $t_{0}$ and $t$ for all values of $p$ and $q$ in the RAP. In the steady state $t_{0} \rightarrow \infty$, we show that $\sigma_{0}^{2}(t)=\lim _{t_{0} \rightarrow \infty} \sigma_{0}^{2}\left(t_{0}, t_{0}+t\right) \sim A_{S R A P} t^{1 / 2}$ for large $t$ for the symmetric RAP (SRAP) with $p=q$. For the asymmetric RAP (ARAP) where $p>q$, we find $\sigma_{0}^{2}(t) \sim D_{A R A P} t$ for large $t$. The constants $A_{S R A P}=2 \rho^{-2}\left(p \mu_{1} / \pi\right)^{1 / 2} \mu_{2} /\left(\mu_{1}-\mu_{2}\right)$ and $D_{A R A P}=\rho^{-2}(p-q) \mu_{1} \mu_{2} /\left(\mu_{1}-\mu_{2}\right)$ are computed exactly. For the special case $q=0$ and $p=1$, $D_{A R A P}$ reduces to $D_{1}$ computed earlier in Refs. 4,20.

2. We compute exactly the universal scaling function that describes the crossover behavior of $\sigma_{0}^{2}(t)$ from the subdiffusive $t^{1 / 2}$ growth to the diffusive $t$ growth as one switches on an infinitesimal bias $(p-q)$.

3. We generalize the single tracer particle fluctuation $\sigma_{0}^{2}\left(t_{0}, t_{0}+t\right)$ to the fluctuation in the position difference of two tagged particles defined as $\sigma_{r}^{2}\left(t_{0}, t_{0}+\right.$ $t)=\left\langle\left[\zeta_{i+r}\left(t_{0}+t\right)-\zeta_{i}\left(t_{0}\right)\right]^{2}\right\rangle$. We show that in the steady state $\sigma_{r}^{2}(t)=\lim _{t_{0} \rightarrow \infty} \sigma_{r}^{2}\left(t_{0}, t_{0}+t\right)$ grows monotonically with $t$ for a fixed tag shift $r$ for the SRAP. For the ARAP on the other hand, it grows with $t$ in a non-monotonic fashion with a single minimum at a characteristic time $t^{*}=r / \mu_{1}(p-q)$.

4. We also compute various scaling functions that describe the crossover of the tracer fluctuations from their non-steady state behavior to the steady state behavior as the waiting time $t_{0} \rightarrow \infty$.

The paper is organized as follows. In Sec. II, we define the model precisely and set up our notations. In Sec. III, we calculate the equal time correlation function for the RAP for all $p$ and $q$. Sec. IV contains the exact calculation of the unequal time correlation function. In Sec. $\mathrm{V}$ we compute the mean squared fluctuation in the displacement of a single tracer particle. The Secs. VA and VB contain respectively the discussions on the SRAP and the ARAP, while the crossover between them is discussed in Sec. VC. The Sec. VI contains the generalization to the two-tag correlation functions. Finally we conclude with a summary and discussion in Sec. VII.

\section{THE MODEL AND PRELIMINARIES}

We consider a system of particles of average density $\rho$ located on a real line. Let $x_{i}(t)$ denote the position of the $i$-th particle at time $t$ (see Fig. 1). In an infinitesimal time interval $d t$, each particle jumps with probability $p d t$ to the right, with probability $q d t$ to the left and with probability $1-(p+q) d t$ it rests at its original location. The actual amount by which a particle jumps (either to the right or to the left) is a random fraction of the gap between the particle and its neighboring particle (to the right or to the left). For example, the jump to the right is by an amount $r_{i}^{+}\left(x_{i+1}-x_{i}\right)$ and to the left by $r_{i}^{-}\left(x_{i}-x_{i-1}\right)$. The random variables $r_{i}^{ \pm}$are independently drawn from the interval $[0,1]$ and each is distributed according to the same pdf $f(r)$ which is arbitrary. We start from an arbitrary but fixed initial condition at $t=0$ and averaging of physical quantities is done over all histories of evolution keeping the initial condition fixed. The time evolution of the positions $x_{i}(t)$ 's can be represented by the exact Langevin equation

$$
x_{i}(t+d t)=x_{i}(t)+\gamma_{i}(t),
$$

where $\gamma_{i}(t)$ are random variables given by 


$$
\gamma_{i}(t)=\left\{\begin{array}{l}
r_{i}^{+}\left(x_{i+1}(t)-x_{i}(t)\right) \text { with prob } p d t \\
r_{i}^{-}\left(x_{i-1}(t)-x_{i}(t)\right) \text { with prob } q d t \\
0 \quad \text { with prob } 1-(p+q) d t .
\end{array}\right.
$$

The random variables $r_{i}^{ \pm}$are independent and each is distributed over the interval $[0,1]$ with the same pdf $f(r)$. The $k$-th moment of the pdf is denoted by $\mu_{k}=$ $\int_{0}^{1} d r r^{k} f(r)$. Note that since $0 \leq r \leq 1$ and $f(r) \geq 0$, $\mu_{1} \geq \mu_{2}$.

We define a new random variable $\zeta_{i}(t)$ which measures the deviation of $x_{i}(t)$ from its mean value

$$
\zeta_{i}(t)=x_{i}(t)-\left\langle x_{i}(t)\right\rangle .
$$

From Eqs. (11) and (2), one can easily derive the evolution rules for the $\zeta_{i}$ variables. We find

$$
\zeta_{i}(t+d t)=\zeta_{i}(t)-(p-q) \frac{\mu_{1}}{\rho} d t+\eta_{i}(t),
$$

where $\eta_{i}(t)$ is given by

$\eta_{i}(t)=\left\{\begin{array}{lr}r_{i}^{+}\left(\zeta_{i+1}(t)-\zeta_{i}(t)+\rho^{-1}\right) & \text { with prob pdt } \\ r_{i}^{-}\left(\zeta_{i-1}(t)-\zeta_{i}(t)-\rho^{-1}\right) & \text { with prob } q d t \\ 0 & \text { with prob } 1-(p+q) d t .\end{array}\right.$

By definition, $\left\langle\zeta_{i}(t)\right\rangle=0$. Also from Eq. (5), it follows that $\left\langle\eta_{i}(t)\right\rangle=(p-q) \frac{\mu_{1}}{\rho} d t$.

In this paper, we will focus on the mean squared displacement of a tagged particle. It turns out that the asymptotic behavior of the mean squared displacement depends crucially on whether one starts measuring these fluctuations after some finite waiting time $t_{0}$ or if one first waits for an infinite time and then starts measuring the statistics. The latter corresponds to measuring the fluctuations in the steady state. This is similar to the 'approach to stationary' versus 'stationary' regimes found in various interface models 21. This can be quantified precisely in terms of the following correlation function,

$$
\begin{aligned}
\sigma_{0}^{2}\left(t_{0}, t_{0}+t\right) & =\left\langle\left(\zeta_{i}\left(t+t_{0}\right)-\zeta_{i}\left(t_{0}\right)\right)^{2}\right\rangle, \\
& =G_{0}\left(t+t_{0}\right)+G_{0}\left(t_{0}\right)-2 C_{0}\left(t_{0}, t_{0}+t\right),
\end{aligned}
$$

where $G_{r}(t)=\left\langle\zeta_{i}(t) \zeta_{i+r}(t)\right\rangle$ is the equal time correlation function and $C_{r}\left(t_{0}, t_{0}+t\right)=\left\langle\zeta_{i}\left(t_{0}\right) \zeta_{i+r}\left(t_{0}+t\right)\right.$ with $t>0$ denotes the unequal time correlation function. For $t=0$, the unequal time correlation function reduces to the equal time correlation function, $C_{r}\left(t_{0}, t_{0}\right)=G_{r}\left(t_{0}\right)$. Note that we have assumed an infinite system so that the translational invariance holds. In the next two sections we calculate analytically the correlation functions $G_{r}(t)$ and $C_{r}\left(t_{0}, t_{0}+t\right)$ respectively.

\section{EQUAL TIME CORRELATION FUNCTION}

In this section, we calculate the equal time correlation function $G_{r}(t)=\left\langle\zeta_{i}(t) \zeta_{i+r}(t)\right\rangle$ exactly for the RAP for all $p$ and $q$. Our starting point is Eq. (位) in conjunction with Eq. (5) describing the evolution of the $\zeta_{i}$ variables with time. We consider the evolution equations (Eq. (4)) for both $\zeta_{i}(t+d t)$ and $\zeta_{i+r}(t+d t)$, multiply them and then take the average \langle\rangle over all histories, keeping terms only upto $O(d t)$. This yields, in the limit $d t \rightarrow 0$, the exact evolution equation of the correlation function $G_{r}(t)$ and we obtain,

$$
\begin{aligned}
\frac{d}{d t} G_{r}(t) & =\mu_{1}(p+q)\left[G_{r+1}(t)+G_{r-1}(t)-2 G_{r}(t)\right] \\
& +\delta_{r, 0} \mu_{2}(p+q)\left[\rho^{-2}+2\left(G_{0}(t)-G_{1}(t)\right)\right] .
\end{aligned}
$$

The Eq. (8) is valid for all positive and negative integers $r$ including $r=0$ and clearly $G_{r}(t)=G_{-r}(t)$. Thus the equation of evolution for the two point correlations involve only two point correlations and not higher order correlations. This closure property is crucial for obtaining an exact solution for the correlation functions. The key reason behind this closure lies in the fact that the random fractions $r_{i}^{ \pm}$'s at time $t$ are independent of the $\zeta_{i}(t)$. One noteworthy fact about Eq. (\&) is that the rates $p$ and $q$ make their appearance only as an overall multiplicative factor $(p+q)$. We could absorb this factor into the time by doing a suitable rescaling, and hence, the equal time correlation function $G_{r}(t)$ is same for both the ARAP and the SRAP.

We note that this equation was also derived in Ref. 201 by a rather lengthy method, but was left unsolved. In this section, we derive an exact solution of Eq. (8). Note that even though Eq. (8) represents the diffusion equation (in discrete space) with a source term at the origin $r=0$, its solution is nontrivial due to the fact that the source term depends on $G_{0}(t)$ and $G_{1}(t)$ which need to be determined self-consistently. Similar diffusion equations with source term for the correlation functions have also appeared recently in the context of aggregation models with injection [22]. Before proceeding to solve Eq. (8), we first set up our notations. We define the standard Fourier transform

$$
\bar{G}(k, t)=\sum_{r=-\infty}^{\infty} G_{r}(t) e^{i k r},
$$

the Laplace transform

$$
\tilde{G}_{r}(s)=\int_{0}^{\infty} G_{r}(t) e^{-s t} d t,
$$

and the joint Fourier-Laplace transform,

$$
F(k, s)=\int_{0}^{\infty} \bar{G}(k, t) e^{-s t} d t=\sum_{r=-\infty}^{\infty} \tilde{G}_{r}(s) e^{i k r} .
$$

Taking the joint Fourier-Laplace transform of Eq. (8) we obtain 


$$
F(k, s)=\frac{\mu_{2}(p+q)\left[\rho^{-2}+2 s\left(\tilde{G}_{0}(s)-\tilde{G}_{1}(s)\right)\right]}{s\left[s+2 \mu_{1}(p+q)(1-\cos k)\right]},
$$

where we have assumed that initially $G_{r}(0)=0$ which is true for any fixed initial condition. For random initial condition, $F(k, s)$ will contain additional terms arising from the initial condition, but one can show that they do not contribute to the asymptotic large time properties of $G_{r}(t)$ as long as the initial condition has only short ranged correlations. We therefore use $G_{r}(0)=0$ without any loss of generality.

The Eq. (12) contains two unknowns $\tilde{G}_{0}(s)$ and $\tilde{G}_{1}(s)$. One of them, say $\tilde{G}_{1}(s)$ can however be expressed in terms of $\tilde{G}_{0}(s)$ by taking directly the Laplace transform of Eq. (8) for $r=0$ and using $G_{1}(t)=G_{-1}(t)$. This gives the relation

$$
s \tilde{G}_{0}(s)=(p+q)\left[\frac{\mu_{2} \rho^{-2}}{s}-2\left(\mu_{1}-\mu_{2}\right)\left(\tilde{G}_{0}(s)-\tilde{G}_{1}(s)\right)\right] .
$$

Substituting Eq. (13) in Eq. (12) we obtain

$$
F(k, s)=\frac{\mu_{2}}{\left(\mu_{1}-\mu_{2}\right)} \frac{\left[\mu_{1}(p+q) \rho^{-2}-s^{2} \tilde{G}_{0}(s)\right]}{s\left[s+2 \mu_{1}(p+q)(1-\cos k)\right]} .
$$

We now have to determine $\tilde{G}_{0}(s)$ self-consistently. This can be done by using the inverse Fourier transform

$$
\tilde{G}_{r}(s)=\frac{1}{2 \pi} \int_{-\pi}^{\pi} F(k, s) e^{-i k r} d k .
$$

Substituting the expression of $F(k, s)$ from Eq. (14) in Eq. (15) at $r=0$, we obtain the exact $\tilde{G}_{0}(s)$

$$
\tilde{G}_{0}(s)=\frac{\mu_{1} \mu_{2}(p+q)}{\left(\mu_{1}-\mu_{2}\right)} \frac{\rho^{-2} I(0, s)}{s\left[1+\frac{\mu_{2}}{\left(\mu_{1}-\mu_{2}\right)} s I(0, s)\right]},
$$

where $I(r, s)$ is given by the integral

$$
\begin{aligned}
I(r, s) & =\frac{1}{2 \pi} \int_{-\pi}^{\pi} \frac{e^{-i k r} d k}{\left[s+2 \mu_{1}^{\prime}(1-\cos k)\right]} \\
& =\frac{1}{\sqrt{s^{2}+4 \mu_{1}^{\prime} s}}\left(\frac{2 \mu_{1}^{\prime}+s-\sqrt{s^{2}+4 \mu_{1}^{\prime} s}}{2 \mu_{1}^{\prime}}\right)^{|r|},
\end{aligned}
$$

where $\mu_{1}^{\prime}=\mu_{1}(p+q)$. Knowing $\tilde{G}_{0}(s)$ determines $F(k, s)$ completely by Eq. (14) and hence $\tilde{G}_{r}(s)$ for all $r$ by the Fourier inversion formula in Eq. (15). We obtain

$$
\tilde{G}_{r}(s)=\frac{\mu_{1} \mu_{2}(p+q)}{\left(\mu_{1}-\mu_{2}\right)} \frac{\rho^{-2} I(r, s)}{s\left[1+\frac{\mu_{2}}{\left(\mu_{1}-\mu_{2}\right)} s I(0, s)\right]},
$$

where $I(r, s)$ is given by Eq. (17).
To obtain $G_{r}(t)$ we need to perform the inverse Laplace transform $G_{r}(t)=\mathcal{L}^{-1}\left[\tilde{G}_{r}(s)\right]$ with respect to $s$. In general for arbitrary $t$ this is difficult. However, for large $t$, this inverse can be obtained in closed form. For large $t$, one needs to consider the small $s$ behavior of $\tilde{G}_{r}(s)$ in Eq. (18). Let us first consider the case $r=0$. Putting $r=0$ in Eq. (17) and taking the $s \rightarrow 0$ limit we find to leading order,

$$
I(0, s) \sim \frac{1}{2 \sqrt{\mu_{1}(p+q) s}} .
$$

Substituting this small $s$ expression of $I(0, s)$ in Eq. (16) and taking the inverse Laplace transform we find that to leading order for large $t$,

$$
G_{0}(t)=\frac{\sqrt{\mu_{1}(p+q)} \mu_{2} \rho^{-2}}{\left(\mu_{1}-\mu_{2}\right) \sqrt{\pi}} \sqrt{t} .
$$

Next we consider the behavior of $G_{r}(t)$ for $|r|>0$. From Eq. (17) it is clear that the appropriate scaling limit consists of taking the limit $s \rightarrow 0,|r| \rightarrow \infty$ but keeping $|r| \sqrt{s}$ fixed. In this scaling limit, Eq. (17) yields,

$$
I(r, s)=\frac{1}{2 \sqrt{\mu_{1}(p+q) s}} \exp \left(\frac{-|r| \sqrt{s}}{\sqrt{\mu_{1}(p+q)}}\right) .
$$

We note that the formula for $I(r, s)$ in Eq. (21) reduces to Eq. (19) for $|r|=0$. This indicates that even though Eq. (21) was derived in the scaling limit, it continues to hold even for $r=0$.

Substituting this small $s$ expression of $I(r, s)$ in Eq. (18) and taking the inverse Laplace transform we obtain for large $t$,

$$
G_{r}(t)=\frac{\sqrt{\mu_{1}(p+q)} \mu_{2} \rho^{-2}}{2\left(\mu_{1}-\mu_{2}\right)} \mathcal{L}^{-1}\left[s^{-3 / 2} e^{\left.-|r| \sqrt{s /\left[\mu_{1}(p+q)\right]}\right]} .\right.
$$

Fortunately the inverse Laplace transform in Eq. (22) can be done in closed form which gives us the following asymptotic scaling behavior of the equal time correlation function $G_{r}(t)$,

$$
G_{r}(t)=\frac{\sqrt{\mu_{1}(p+q)} \mu_{2} \rho^{-2}}{\left(\mu_{1}-\mu_{2}\right) \sqrt{\pi}} \sqrt{t} f_{1}\left(\frac{|r|}{2 \sqrt{\mu_{1}(p+q) t}}\right) .
$$

Here $f_{1}(y)$ is a universal scaling function independent of the model parameters such as $p, q$ and the moments $\mu_{k}$ of the pdf $f(r)$ and is given by

$$
f_{1}(y)=e^{-y^{2}}-\sqrt{\pi} y \operatorname{erfc}(y),
$$

where $\operatorname{erfc}(y)=2 / \sqrt{\pi} \int_{y}^{\infty} e^{-u^{2}} d u$ is the standard complimentary error function. This scaling function has the asymptotic behaviors, $f_{1}(y) \sim 1-\sqrt{\pi} y$ as $y \rightarrow 0$ and $\sim y^{-2} e^{-y^{2}} / 2$ for $y \rightarrow \infty$. 
As a final remark, we note again that if one puts $|r|=0$ in the formula for $G_{r}(t)$ in Eq. (23) one recovers the correct $G_{0}(t)$ as given by Eq. (20). Thus the scaling range includes even the $r=0$ point. The Eq. (20) thus provides us the exact behavior of the first two terms in the expression for $\sigma_{0}^{2}\left(t_{0}, t_{0}+t\right)$ in Eq. (7). The remaining task is to evaluate the third term in Eq. (7) which involves the unequal time correlation function and this is done in the next section.

\section{UNEQUAL TIME CORRELATIONS}

In this section we compute the two time tag-tag correlation function $C_{r}\left(t_{0}, t_{0}+t\right)=\left\langle\zeta_{i}\left(t_{0}\right) \zeta_{i+r}\left(t_{0}+t\right)\right\rangle$ for the RAP. We start at time $t_{0}$ and then evolve the $\zeta_{i+r}$ variables by Eq. (4) for all subsequent time. Let us first rewrite the Eq. (幽 at time $t_{0}+t+d t$,

$$
\zeta_{i+r}\left(t_{0}+t+d t\right)=\zeta_{i+r}\left(t_{0}+t\right)-(p-q) \frac{\mu_{1}}{\rho} d t+\eta_{i+r}\left(t_{0}+t\right) .
$$

We then multiply both sides of Eq. (25) by $\zeta_{i}\left(t_{0}\right)$ and average over the noise keeping terms only upto $O(d t)$. In the limit $d t \rightarrow 0$, we obtain the exact evolution equation of the two time correlation function,

$$
\begin{aligned}
\frac{d C_{r}\left(t_{0}, t_{0}+t\right)}{d t} & =\mu_{1}\left[p C_{r+1}\left(t_{0}, t_{0}+t\right)+q C_{r-1}\left(t_{0}, t_{0}+t\right)\right. \\
& \left.-(p+q) C_{r}\left(t_{0}, t_{0}+t\right)\right] \quad \text { for } t \geq 0 .
\end{aligned}
$$

Note that at $t=0$, the unequal time correlation function reduces to the equal time correlation function $C_{r}\left(t_{0}, t_{0}\right)=G_{r}\left(t_{0}\right)$. Thus starting at $t=0$ with the initial condition $C_{r}\left(t_{0}, t_{0}\right)=G_{r}\left(t_{0}\right)$, the function $C_{r}\left(t_{0}, t_{0}+t\right)$ evolves with time $t$ according to the Eq. (26).

As in the preceding section, we define the Fourier transform $\bar{C}\left(k, t_{0}, t_{0}+t\right)=\sum_{r=-\infty}^{\infty} C_{r}\left(t_{0}, t_{0}+t\right) e^{i k r}$. Taking the Fourier transform of Eq. (26) we obtain

$$
\bar{C}\left(k, t_{0}, t_{0}+t\right)=\bar{G}\left(k, t_{0}\right) e^{-\mu_{1} \alpha(k) t},
$$

where $\alpha(k)=p+q-\left(p e^{-i k}+q e^{i k}\right)$ and $\bar{G}\left(k, t_{0}\right)$ is the Fourier transform of the equal time correlation function as defined by Eq. (9). Taking further the Laplace transform $H(k, s, t)=\int_{0}^{\infty} \bar{C}\left(k, t_{0}, t_{0}+t\right) e^{-s t_{0}} d t_{0}$ of Eq. (27) we obtain

$$
H(k, s, t)=F(k, s) e^{-\mu_{1} \alpha(k) t},
$$

where $F(k, s)$ is given exactly by Eq. (14) with $\tilde{G}_{0}(s)$ determined from Eq. (16).

Proceeding as in the previous section, the Laplace transform $\tilde{C}_{r}(s, t)=\int_{0}^{\infty} C_{r}\left(t_{0}, t_{0}+t\right) e^{-s t_{0}} d t_{0}$ can then be determined from the joint Fourier-Laplace transform $H(k, s, t)$ by the inversion formula

$$
\tilde{C}_{r}(s, t)=\frac{1}{2 \pi} \int_{-\pi}^{\pi} H(k, s, t) e^{-i k r} d k,
$$

where $H(k, s, t)$ is given by Eq. (28). Substituting in Eq. (29) the exact expression of $F(k, s)$ from Eq. (14) and that of $\tilde{G}_{0}(s)$ from Eq. (16), we obtain the following final expression of the Laplace transform

$$
\begin{aligned}
\tilde{C}_{r}(s, t) & =\frac{\mu_{1} \mu_{2}(p+q)}{\left(\mu_{1}-\mu_{2}\right)} \frac{\rho^{-2}}{s\left[1+\frac{\mu_{2}}{\left(\mu_{1}-\mu_{2}\right)} s I(0, s)\right]} \\
& \times \frac{1}{2 \pi} \int_{-\pi}^{\pi} \frac{e^{-i k r-\mu_{1} \alpha(k) t} d k}{\left[s+2 \mu_{1}(p+q)(1-\cos k)\right]} .
\end{aligned}
$$

Note that for $t=0, \tilde{C}_{r}(s, t)$ as given by Eq. (30) reduces to $\tilde{G}_{r}(s)$ given by Eq. (18) as expected. The equation (30) is central to our subsequent analysis for various limiting behaviors.

\section{MEAN SQUARED TRACER AUTO-FLUCTUATION}

In this section we calculate $\sigma_{0}^{2}\left(t_{0}, t_{0}+t\right)$ in the RAP using the exact results for the equal time and two time correlation functions obtained in the previous sections. We consider first the symmetric case SRAP with $p=q$ in subsection A followed by the derivation for the asymmetric case ARAP with $p>q$ in subsection B. In subsection $\mathrm{C}$, we show how the steady state fluctuation $\sigma_{0}^{2}(t)=\lim _{t_{0} \rightarrow \infty} \sigma_{0}^{2}\left(t_{0}, t_{0}+t\right)$ crosses over from the subdiffusive behavior to the diffusive behavior as one switches on an infinitesimal bias and we calculate the crossover scaling function exactly.

\section{A. SRAP}

Here we consider the symmetric case $p=q$. For the calculation of $\sigma_{0}^{2}\left(t_{0}, t_{0}+t\right)$ we only need the asymptotic behavior of $C_{r}\left(t_{0}, t_{0}+t\right)$ for $r=0$ as evident from Eq. (7). To obtain $C_{0}\left(t_{0}, t_{0}+t\right)$ we need to invert the Laplace transform in Eq. (30) for $r=0$ and $p=q$. As before, this inversion is difficult in general for all $t_{0}$. However the finite but large $t_{0}$ limit can be worked out by analyzing the small $s$ behavior of Eq. (30). It turns out that the appropriate scaling limit in this case involves taking $s \rightarrow 0, t \rightarrow \infty$ but keeping st fixed. In this scaling limit, the integration in Eq. (30) can be carried out in closed form and we obtain (with $p=q$ ),

$$
\tilde{C}_{0}(s, t)=\frac{\sqrt{2 p \mu_{1}} \mu_{2} \rho^{-2}}{2\left(\mu_{1}-\mu_{2}\right) s^{3 / 2}} e^{s t / 2} \operatorname{erfc}(\sqrt{s t / 2}) .
$$

We then need to invert the Laplace transform in Eq. (31) with respect to $s$ to obtain the asymptotic behavior of 
$C_{0}\left(t_{0}, t_{0}+t\right)$ for large $t_{0}$. Fortunately this inversion can be done in closed form and we obtain

$$
C_{0}\left(t_{0}, t_{0}+t\right)=\frac{\sqrt{2 p \mu_{1}} \mu_{2} \rho^{-2}}{\left(\mu_{1}-\mu_{2}\right) \sqrt{\pi}} \sqrt{t_{0}} f_{2}\left(\frac{t}{2 t_{0}}\right),
$$

where the scaling function $f_{2}(y)$ is again universal and is given by,

$$
f_{2}(y)=\sqrt{1+y}-\sqrt{y} .
$$

We are now ready to compute $\sigma_{0}^{2}\left(t_{0}, t_{0}+t\right)$ from Eq. (7). Using the result for the equal time correlation in Eq. (20) and the one for the two time correlation in Eq. (32), we obtain from Eq. (7) our main result

$$
\begin{aligned}
& \sigma_{0}^{2}\left(t_{0}, t_{0}+t\right)= \\
& \quad \frac{\sqrt{2 p \mu_{1}} \mu_{2} \rho^{-2}}{\left(\mu_{1}-\mu_{2}\right) \sqrt{\pi}}\left[\sqrt{t_{0}+t}+\sqrt{t_{0}}-2 \sqrt{t_{0}} f_{2}\left(\frac{t}{2 t_{0}}\right)\right],
\end{aligned}
$$

where $f_{2}(y)$ is given by Eq. (33). Note that this result in Eq. (34) is derived in the scaling limit when both $t_{0}$ and $t$ are large with their ratio $t / t_{0}$ kept fixed.

We now discuss two different limits of Eq. (34). First we consider the steady state limit $t_{0} \rightarrow \infty$ with $t$ large but fixed. In this limit, Eq. (34) yields

$$
\sigma_{0}^{2}(t)=\lim _{t_{0} \rightarrow \infty} \sigma_{0}^{2}\left(t_{0}, t_{0}+t\right)=\frac{2 \sqrt{p \mu_{1}} \mu_{2} \rho^{-2}}{\left(\mu_{1}-\mu_{2}\right) \sqrt{\pi}} \sqrt{t} .
$$

In the opposite limit, when the waiting time $t_{0}$ is finite (away from the steady state) but the evolved time $t$ goes to infinity, we obtain from Eq. (34,

$$
\lim _{t \rightarrow \infty} \sigma_{0}^{2}\left(t_{0}, t_{0}+t\right)=\frac{\sqrt{2 p \mu_{1}} \mu_{2} \rho^{-2}}{\left(\mu_{1}-\mu_{2}\right) \sqrt{\pi}} \sqrt{t} .
$$

Thus the mean squared auto-fluctuation in these two opposing limits differ by a factor $\sqrt{2}$. The Eqs. (34), (35) and (36) are amongst the important new results of this paper.

\section{B. ARAP}

In this subsection we calculate $\sigma_{0}^{2}\left(t_{0}, t_{0}+t\right)$ in the asymmetric case when $p>q$. Once again we have to invert the Laplace transform in Eq. (30) for $r=0$ but now with $p>q$. In this case it turns out the appropriate scaling limit consists of taking $s \rightarrow 0, t \rightarrow \infty$ as in the SRAP but keeping $\sqrt{s} t$ instead of the scaling variable $s t$ in the SRAP. In this scaling limit, the integration in Eq. (30) with $r=0$ yields

$$
\tilde{C}_{0}(s, t)=\frac{\sqrt{\mu_{1}(p+q)} \mu_{2} \rho^{-2}}{2\left(\mu_{1}-\mu_{2}\right) s^{3 / 2}} e^{-(p-q) \sqrt{\mu_{1} s /(p+q)} t} .
$$

The Laplace transform in Eq. (37) can be inverted as in Eq. (22) and we obtain
$C_{0}\left(t_{0}, t_{0}+t\right)=\frac{\sqrt{\mu_{1}(p+q)} \mu_{2} \rho^{-2}}{\left(\mu_{1}-\mu_{2}\right) \sqrt{\pi}} \sqrt{t_{0}} f_{1}\left[\frac{\sqrt{\mu_{1}}(p-q) t}{2 \sqrt{(p+q) t_{0}}}\right]$,

where the universal scaling function $f_{1}(y)=e^{-y^{2}}-$ $\sqrt{\pi} y \operatorname{erfc}(y)$ is the same as in Eq. (24).

Substituting the results in Eq. (38) and Eq. (20) in Eq. (7) we obtain

$$
\begin{aligned}
& \sigma_{0}^{2}\left(t_{0}, t_{0}+t\right)=\frac{\sqrt{\mu_{1}(p+q)} \mu_{2} \rho^{-2}}{\left(\mu_{1}-\mu_{2}\right) \sqrt{\pi}} \\
& \quad \mathrm{x}\left[\sqrt{t_{0}+t}+\sqrt{t_{0}}-2 \sqrt{t_{0}} f_{1}\left(\frac{\sqrt{\mu_{1}}(p-q) t}{2 \sqrt{(p+q) t_{0}}}\right)\right] .
\end{aligned}
$$

As in the SRAP we now discuss the two different limits. In the steady state $t_{0} \rightarrow \infty$ with fixed large $t$ we obtain from Eq. (39),

$$
\sigma_{0}^{2}(t)=\lim _{t_{0} \rightarrow \infty} \sigma_{0}^{2}\left(t_{0}, t_{0}+t\right)=\frac{\mu_{1} \mu_{2} \rho^{-2}(p-q)}{\left(\mu_{1}-\mu_{2}\right)} t .
$$

Thus in this case $\sigma_{0}^{2}(t)$ grows diffusively for large $t$, $\sigma_{0}^{2}(t)=D_{A R A P} t$ where the diffusion constant,

$$
D_{A R A P}=\rho^{-2}(p-q) \frac{\mu_{1} \mu_{2}}{\left(\mu_{1}-\mu_{2}\right)},
$$

depends explicitly on $p$ and $q$. For $q=0$ and $p=1$, it reduces to the expression $D_{1}=\rho^{-2} \mu_{1} \mu_{2} /\left(\mu_{1}-\mu_{2}\right)$ derived by Krug and Garcia using the independent jump approximation [4] and later rederived by Schütz [20] using a different approach.

We make a brief comment here on the approach used in Ref. [20] in deriving the diffusion constant $D_{1}$. In his approach, Schütz started with the evolution equation (8) for the equal time correlation function and then used a chain of arguments to derive the diffusion constant $D_{1}$. His approach didn't require any knowledge of the two time correlation function or even the solution of the equal time correlation function. As evident from the definition in Eq. (7) that $\sigma_{0}^{2}\left(t_{0}, t_{0}+t\right)$ requires the knowledge of both the equal and the two time correlation functions. Thus it was rather remarkable that the correct value of the diffusion constant for $q=0$ and $p=1$ was recovered in Ref. [20]. However this turns out to be purely fortuitous. Note that the evolution equation (8) is independent of the bias in the system. Thus the approach of Schütz would predict that the diffusion constant is also completely independent of the bias $(p-q)$ and is always given by $D_{1}$ (provided $t$ is scaled by $(p+q)$ ). This is clearly wrong as evident from the exact expression in Eq. (41). In particular for the symmetric case $p=q=1 / 2$, the arguments of Ref. [20] would predict a diffusive growth of 
$\sigma_{0}^{2}(t)$ with the diffusion constant $D_{1}$. This is again incorrect since for $p=q$ the diffusion constant is 0 from Eq. (41) which is consistent with the correct asymptotic subdiffusive growth of $\sigma_{0}^{2}(t)$ as given exactly by Eq. (35). The problem in the derivation of Schütz can be traced back to the fact that his arguments only used equal time correlations (which involve only $(p+q)$ ) and not the two time correlations. The dependence on the bias $(p-q)$ of the diffusion constant $D_{A R A P}$ comes only from the two time correlations. The derivation of Ref. 20] misses this important fact and is rather fortuitous to obtain the correct value $D_{1}$ of the diffusion constant for the special case when $p=1$ and $q=0$.

We end this subsection by discussing the other limit when the system is away from the steady state, i.e. when $t_{0}$ is large but finite and $t \rightarrow \infty$. In this limit, we obtain from Eq. (39)

$$
\lim _{t \rightarrow \infty} \sigma_{0}^{2}\left(t_{0}, t_{0}+t\right)=\frac{\sqrt{\mu_{1}(p+q)} \mu_{2} \rho^{-2}}{\left(\mu_{1}-\mu_{2}\right) \sqrt{\pi}} \sqrt{t},
$$

the same result as in the SRAP in this limit [Eq. (36)]. Thus away from the steady state the tracer particle doesn't sense the presence of bias. The exact result in Eq. (42) is consistent with that of Krug and Garcia using a phenomenological hydrodynamic equation [4].

\section{Crossover Between SRAP and ARAP}

In the previous subsections, we have seen that the asymptotic large $t$ behavior of $\sigma_{0}^{2}\left(t_{0}, t_{0}+t\right)$ does not depend on the bias $(p-q)$, when the system is away from the steady state (finite $t_{0}$ ). However, in the steady state $\left(t_{0} \rightarrow \infty\right)$ it behaves rather differently in the symmetric and asymmetric cases. In the steady state of the $\operatorname{SRAP}(p=q), \sigma_{0}^{2}(t) \sim t^{1 / 2}$ while for the $\operatorname{ARAP}(p>q)$, $\sigma_{0}^{2}(t) \sim t$. Thus a natural question is: How does the behavior of $\sigma_{0}^{2}(t)$ crosses over from the subdiffusive growth for $p=q$ to the diffusive growth as one switches on an infinitesimal bias $(p-q)$ ? In this subsection we compute exactly the universal scaling function that describes this crossover behavior of $\sigma_{0}^{2}(t)$.

To calculate the crossover behavior we return to our central equation (30) with $r=0$. We have seen in the previous subsections that in the scaling limit $s \rightarrow 0$ and $t \rightarrow \infty$ of the Eq. (30), the appropriate scaling variable that is kept fixed is $s t$ for $p=q$, where as, it is $\sqrt{s} t$ for $p>q$. Thus, to compute the crossover behavior, we need to keep the leading order terms in both of these scaling variables fixed while expanding Eq. (30) for small $s$ and large $t$. This makes the calculation of the crossover behavior somewhat delicate. To leading order, we find after elementary algebra

$$
\tilde{C}_{0}(s, t)=\frac{\sqrt{\mu_{1}(p+q)} \mu_{2} \rho^{-2}}{\left(\mu_{1}-\mu_{2}\right) s^{3 / 2}}
$$

$$
\mathrm{x} \frac{1}{2 \pi} \int_{-\infty}^{\infty} \frac{e^{-i(p-q) \sqrt{\mu_{1} s /(p+q)} t z-s t z^{2} / 2}}{1+z^{2}} d z .
$$

Note that for the symmetric case $p=q$, the integral in Eq. (43) can be done and we get back Eq. (31) of Sec. VA. Similarly, for the asymmetric case $p>q$, in the limit $s \rightarrow 0$ keeping the scaling variable $\sqrt{s} t$ fixed, one drops the second term in the exponential in the integrand of Eq. (43) and performing the resulting integral we recover the Eq. (37) of Sec. VB.

To compute the crossover behavior we need to keep both the terms inside the exponential in the integrand of Eq. (43) and perform the integral. Fortunately this integral can be done in closed form using the standard convolution theorem. We omit the details here and present only the final result,

$$
\begin{aligned}
\tilde{C}_{0}(s, t) & =\frac{\sqrt{\mu_{1}(p+q)} \mu_{2} \rho^{-2}}{4\left(\mu_{1}-\mu_{2}\right) s^{3 / 2}} \\
\mathrm{x} & {\left[e^{u-v} \operatorname{erfc}\left(\frac{2 u-v}{2 \sqrt{u}}\right)+e^{u+v} \operatorname{erfc}\left(\frac{2 u+v}{2 \sqrt{u}}\right)\right], }
\end{aligned}
$$

where $u=s t / 2$ and $v=(p-q) \sqrt{\mu_{1} s /(p+q)} t$. We then expand the Eq. (44) further for small $s$ to obtain the steady state $t_{0} \rightarrow \infty$ behavior. Note that we needed to first do the integral in Eq. (43) and then take the $s \rightarrow 0$ limit. The reverse order unfortunately does not work. Expanding Eq. (44) for small $s$, keeping only the leading order terms in $s$ and finally inverting the Laplace transform of the resulting expression we obtain for large $t_{0}$

$$
\begin{aligned}
& C_{0}\left(t_{0}, t_{0}+t\right)=\frac{\sqrt{\mu_{1}(p+q)} \mu_{2} \rho^{-2}}{\left(\mu_{1}-\mu_{2}\right) \sqrt{\pi}} \\
& \times\left[\sqrt{t_{0}}-\sqrt{\frac{t}{2}} e^{-w^{2}(t)}-\frac{\sqrt{\pi \mu_{1}}(p-q) t}{2 \sqrt{p+q}} \operatorname{erf}(w(t))\right],
\end{aligned}
$$

where $w(t)=(p-q) \sqrt{\mu_{1} t /[2(p+q)]}$.

We now use the results from Eq. (45) and Eq. (20) in Eq. (7) and eventually take the strict $t_{0} \rightarrow \infty$ limit to obtain the final form of the steady state auto-fluctuation

$$
\begin{aligned}
\sigma_{0}^{2}(t) & =\lim _{t_{0} \rightarrow \infty} \sigma_{0}^{2}\left(t_{0}, t_{0}+t\right) \\
& =\frac{\mu_{1} \mu_{2}(p-q) \rho^{-2}}{\left(\mu_{1}-\mu_{2}\right)} t Y\left[\frac{(p-q) \sqrt{\mu_{1} t}}{\sqrt{2(p+q)}}\right],
\end{aligned}
$$

where $Y(y)$ is a universal crossover scaling function given by

$$
Y(y)=\operatorname{erf}(y)+\frac{1}{\sqrt{\pi}} \frac{e^{-y^{2}}}{y} .
$$

The scaling function has the asymptotic behavior $Y(y) \sim$ $1 /(\sqrt{\pi} y)$ as $y \rightarrow 0$ and $Y(y) \rightarrow 1$ and $y \rightarrow \infty$. Note that 
for fixed $p-q>0$, if we take the limit $t \rightarrow \infty$ in Eq. (46) (which corresponds to $y \rightarrow \infty$ in the scaling function in Eq. (47)) we recover the result of Eq. (40). Similarly if we take the $p-q \rightarrow 0$ limit for fixed $t$ in Eq. (46) (corresponding to taking $y \rightarrow 0$ limit in the scaling function $Y(y))$, we recover, as expected, the result of Eq. (35) of the symmetric case. Thus the Eq. (46) and the associated scaling function $Y(y)$ in Eq. (47) describes the crossover behavior from the subdiffusive to diffusive growth as one switches on an infinitesimal bias.

\section{GENERALIZATION TO THE TWO-TAG CORRELATION FUNCTION}

So far in this paper we have concentrated only on the mean squared auto-fluctuation of a tracer particle, $\sigma_{0}^{2}\left(t_{0}, t_{0}+t\right)=\left\langle\left(\zeta_{i}\left(t+t_{0}\right)-\zeta_{i}\left(t_{0}\right)\right)^{2}\right\rangle$. A natural generalization of the auto-fluctuation would be to study the two-tag correlation function defined as

$$
\begin{aligned}
\sigma_{r}^{2}\left(t_{0}, t_{0}+t\right) & =\left\langle\left(\zeta_{i+r}\left(t+t_{0}\right)-\zeta_{i}\left(t_{0}\right)\right)^{2}\right\rangle, \\
& =G_{0}\left(t+t_{0}\right)+G_{0}\left(t_{0}\right)-2 C_{r}\left(t_{0}, t_{0}+t\right),
\end{aligned}
$$

where $G_{r}(t)$ and $C_{r}\left(t_{0}, t_{0}+t\right)$ are the usual equal time time and the two time correlation functions already defined and derived in the previous sections. Note that for $r=0$, the two-tag correlation in Eq. (48) reduces to the single tag function $\sigma_{0}^{2}\left(t_{0}, t_{0}+t\right)$.

Of particular interest would be to compute the twotag correlation function in the steady state, i.e. $\sigma_{r}^{2}(t)=$ $\lim _{t_{0} \rightarrow \infty} \sigma_{r}^{2}\left(t_{0}, t_{0}+t\right)$. For the exclusion process this twotag correlation function was first introduced in Ref. 18] and the presence of bias was found to have a dramatic effect on the time dependence of $\sigma_{r}^{2}(t)$ for a fixed $r$. It was found numerically that while in the SEP $\sigma_{r}^{2}(t)$ increases monotonically with $t$ for a fixed tag-shift $r$, in the ASEP $\sigma_{r}^{2}(t)$ has a non-monotonic dependence on $t$ [18]. In the ASEP $\sigma_{r}^{2}(t)$ first decreases with time $t$, becomes a minimum at some characteristic time $t^{*}$ and then starts increasing again. A harmonic model was proposed in Ref. 18 for which $\sigma_{r}^{2}(t)$ could be computed analytically and was found to be in qualitative agreement with the numerical results of the exclusion process. But to the best of our knowledge, exact calculation of $\sigma_{r}^{2}(t)$ for the exclusion process is still an unsolved problem. However it turns out that for the RAP, it is possible to compute this function $\sigma_{r}^{2}(t)$ exactly for large $t$. The exact solution of $\sigma_{r}^{2}(t)$ in the RAP, as shown below, shares the similar features as in the exclusion process.

From Eq. (49) it is evident that we just need to compute the large $t_{0}$ behavior of the two time correlation function $C_{r}\left(t_{0}, t_{0}+t\right)$ for fixed nonzero $r$. In the previous sections we have analyzed in detail the $r=0$ case. It turns out that the analysis for $r \neq 0$ proceeds more or less in the same manner as in the $r=0$ case. We start, once again, from the central equation (30). To avoid separate calculations for the SRAP and the ARAP, we take the line of approach used to calculate the crossover behavior in subsection VC. For $r \neq 0$, it turns out that the equation (43) gets replaced by a similar looking equation,

$$
\begin{aligned}
& \tilde{C}_{r}(s, t)=\frac{\sqrt{\mu_{1}(p+q)} \mu_{2} \rho^{-2}}{\left(\mu_{1}-\mu_{2}\right) s^{3 / 2}} \\
& \times \mathrm{x} \frac{1}{2 \pi} \int_{-\infty}^{\infty} \frac{e^{-i z \sqrt{s /\left[\mu_{1}(p+q)\right]} R-s t z^{2} / 2}}{1+z^{2}} d z,
\end{aligned}
$$

where $R=r+\mu_{1}(p-q) t$ signifies the drift of the particles to the right with average velocity $\mu_{1}(p-q)$ for $p>q$. Clearly for $r=0$, Eq. (50) reduces to Eq. (43). Starting with Eq. (50) we then follow exactly the same steps as used in subsection VC. Since the steps are identical we skip all the details and present only the final result. In the strict steady state limit $t_{0} \rightarrow \infty$, we finally obtain the following scaling form

$$
\begin{aligned}
\sigma_{r}^{2}(t) & =\lim _{t_{0} \rightarrow \infty} \sigma_{r}^{2}\left(t_{0}, t_{0}+t\right) \\
& =\frac{\sqrt{2 \mu_{1}(p+q)} \mu_{2} \rho^{-2}}{\left(\mu_{1}-\mu_{2}\right) \sqrt{\pi}} \sqrt{t} W\left(\frac{R}{\sqrt{2 \mu_{1}(p+q) t}}\right),
\end{aligned}
$$

where $R=r+\mu_{1}(p-q) t$ and $W(y)$ is again a universal scaling function given by,

$$
W(y)=e^{-y^{2}}+\sqrt{\pi} y \operatorname{erf}(y)
$$

Clearly $\mu_{1}(p-q) t$ represents the average drift while $l(t)=\sqrt{2 \mu_{1}(p+q) t}$ represents the diffusive length scale.

We note that the scaling function $W(y)$ is a symmetric function of $y$ about $y=0$ with a minimum at $y=0$. For the SRAP, $p=q$ and hence $R=r$. Thus for a fixed $r$, it follows from Eq. (51) that $\sigma_{r}^{2}(t)$ increases monotonically with $t$. For the ARAP on the other hand, $p>q$ and $R=r+\mu_{1}(p-q) t$. If one fixes $r$ to a negative value and increases $t$, the variable $R$ remains negative till the characteristic time $t=t^{*}=r / \mu_{1}(p-q)$, beyond which it becomes positive. The scaling variable $y=R / \sqrt{2 \mu_{1}(p+q) t}$ behaves in the same way. Thus $\sigma_{r}^{2}(t)$ in Eq. (51) first decreases with time, becomes a minimum at $t=t^{*}=-r / \mu_{1}(p-q)$ and then starts increasing again. In Fig. (2) we plot the function $\sigma_{r}^{2}(t)$ in Eq. (51) for both the SRAP (with $p=q=1 / 2$ ) and the ARAP (with $p=1$ and $q=0$ ) for the same value of $r=-2$ and choosing the parameter values $\mu_{1}=1 / 2$, $\mu_{2}=1 / 4, \rho=1$. These features in the RAP, derived here exactly, are qualitatively similar to those in the exclusion process studied in Ref. [18]. 


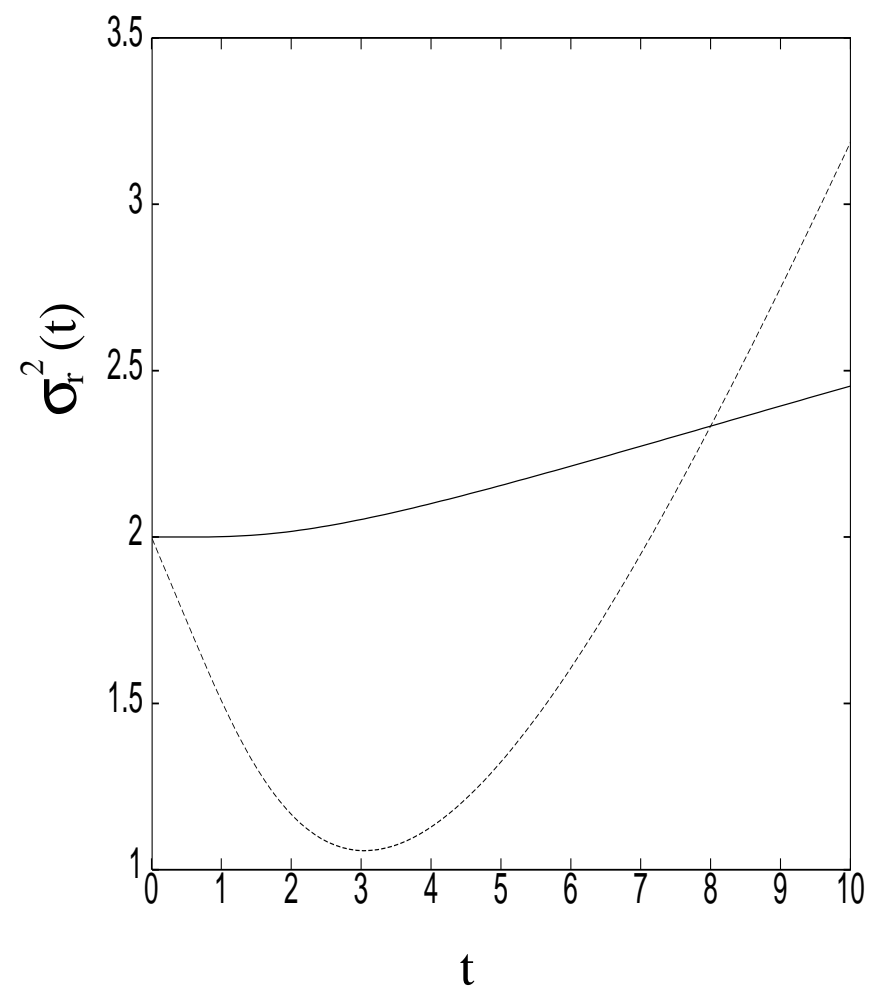

FIG. 2. The steady state two-tag correlation function $\sigma_{r}^{2}(t)$ in Eq. (51) plotted as a function of $t$ for fixed $r=-2$ for parameter values $\mu_{1}=1 / 2, \mu_{2}=1 / 4$ and $\rho=1$. The solid line shows the monotonic growth of $\sigma_{r}^{2}(t)$ with $t$ for the SRAP ( $p=q=1 / 2)$ while the dashed line shows the non-monotonic growth for the ARAP $(p=1$ and $q=0)$.

\section{CONCLUSIONS}

In this paper we have studied analytically the mean squared fluctuations in the diffusion of both a single tagged particle and two tagged particles in the random average process (RAP) for all values of the hopping rates $p$ and $q$ in one dimension. We have shown that in the steady state, the auto-fluctuation of a tagged particle grows subdiffusively as $\sigma_{0}^{2}(t) \sim A_{S R A P} t^{1 / 2}$ for $p=q$ and diffusively $\sigma_{0}^{2}(t) \sim D_{A R A P} t$ for $p>q$ where $A_{S R A P}=2 \rho^{-2}\left(p \mu_{1} / \pi\right)^{1 / 2} \mu_{2} /\left(\mu_{1}-\mu_{2}\right)$ and $D_{A R A P}=$ $\rho^{-2}(p-q) \mu_{1} \mu_{2} /\left(\mu_{1}-\mu_{2}\right)$. These behaviors of $\sigma_{0}^{2}(t)$ are similar to those in the simple exclusion process except the prefactors $A=(2 / \pi)^{1 / 2}(1-\rho) / \rho$ 12 14 and $D=(p-q)(1-\rho)$ 15, 16 are different in the exclusion process. Besides the steady state mean squared two-tag fluctuation $\sigma_{r}^{2}(t)$ in the RAP grows monotonically with $t$ for $p=q$ and non-monotonically for $p>q$, in much the same way as in the exclusion process.

These findings raise the question whether or not the RAP is in the same universality class as the simple exclusion process in one dimension. Perhaps the RAP is just a coarse grained version of the exclusion process in one dimension? The answer to this question seems to be in the negative due to a very crucial difference between the two processes. In the exclusion process for $p>q$, it is well known that there exists an anomalous $t^{2 / 3}$ growth hidden in the problem apart from the usual $t^{1 / 2}$ and $t$ growth [19,17]. This anomalous growth shows up either in the mean squared fluctuation of the center of mass of the particles when viewed from a special moving frame [19] or alternately in the two-tag correlation function $\sigma_{r}^{2}(t)$ if one chooses the tag shift $r$ to be sliding with time with a special velocity $r=-\rho^{2}(p-q)$ 17. It turns out that the prefactor of this $t^{2 / 3}$ growth is proportional to $\propto \frac{d^{2} j(\rho)}{d \rho^{2}}$ where $j(\rho)$ is the current density in a hydrodynamical description 17. For the exclusion process, $j(\rho)=\rho(1-\rho)$ and hence the prefactor is nonzero. For the RAP on the other hand, $j(\rho)=\mu_{1}(p-q)$ and is independent of $\rho$. This is because $j(\rho)=\rho\langle v\rangle$ where the average velocity $\langle v\rangle=\mu_{1}(p-q) / \rho$ as can be easily derived from Eqs. (11) and (2). As a result, for the RAP, the anomalous $t^{2 / 3}$ growth is absent which puts it in a different universality class than the simple exclusion process. In this sense the RAP seems to be closer to the harmonic model studied in Ref. [18].

In this paper we have considered the RAP only in one dimension. An obvious generalization would be to higher dimensions. A natural way to generalize the model to higher dimensions would be as follows. One considers particles located in the continuous $d$-dimensional space. In a small time interval $d t$, each particle makes a list of all its nearest neighbors in various directions in space, chooses one of them at random and jumps in the corresponding direction by a random fraction of the Euclidean distance to that neighbor. This is an isotropic version, a generalization of the SRAP. Similarly one can define an anisotropic version as well. To the best of our knowledge, the RAP has not been studied so far in higher dimensions. The question of tracer diffusion in higher dimensions, especially in two dimensions where one may expect a logarithmic correction, also remains completely open.

[1] T. M. Liggett, Interacting Particle Systems (SpringerVerlag, New York, 1985).

[2] H. Spohn, Large Scale Dynamics of Interacting Particles (Springer-Verlag, Berlin Heidelberg, 1991).

[3] B. Derrida and M.R. Evans, p-277 in Nonequilibrium Statistical Mechanics in one dimension, edited by V. Privman (Cambridge University Press, Cambridge, 1997).

[4] J. Krug and J. Garcia, J. Stat. Phys. 99, 31 (2000).

[5] R. Rajesh and S. N. Majumdar, J. Stat. Phys. 99, 943 (2000).

[6] S. N. Coppersmith, C.-h. Liu, S. N. Majumdar, O. Narayan and T. A. Witten, Phys. Rev. E 53, 4673 (1996). 
[7] Z. A. Melzak, Mathematical Ideas, Modeling and Applications, Vol II of Companion to Concrete Mathematics (Wiley, New York, 1976), p.271.

[8] P. A. Ferrari and L. R. G. Fontes, El. J. Prob. 3, Paper no. 6 (1998).

[9] S. Ispolatov, P. L. Krapivsky and S. Redner, Eur. Phys. J. B2, 267 (1998).

[10] D. Aldous and P. Diaconis, Probablity Theory and Related Fields, 103, 199 (1995).

[11] F. Zielen and A. Schadschneider, cond-mat/0104298.

[12] T. Harris, J. Appl. Prob., 2, 323 (1965)

[13] R. Arratia, Ann. Prob., 11, 362 (1983).

[14] S. Alexander and P. Pincus, Phys. Rev. B. 18, 2011 (1978).

[15] A. Demasi and P. A. Ferrari, J. Stat. Phys., 38, 603 (1985).
[16] R. Kutner and H. van Beijeren, J. Stat. Phys., 39, 317 (1985).

[17] S. N. Majumdar and M. Barma, Physica A. 177, 366 (1991).

[18] S. N. Majumdar and M. Barma, Phys. Rev. B. 44, 5306 (1991).

[19] H. van Beijeren, R. Kutner, and H. Spohn, Phys. Rev. Lett. 54, 2026 (1985).

[20] G. M. Schütz, J. Stat. Phys. 99, 1045 (2000).

[21] J. Krug, H. Kallabis, S.N. Majumdar, S.J. Cornell, A.J. Bray, and C. Sire, Phys. Rev. E 56, 2702 (1997); S.N. Majumdar and A.J. Bray, cond-mat/0009439, to appear in Phys. Rev. Lett.

[22] R. Rajesh and S. N. Majumdar, Phys. Rev. E. 62, 3186 (2000). 